\title{
Correction to: False-positive fecal immunochemical test results in colorectal cancer screening and gastrointestinal drug use
}

\author{
M. J. Domper Arnal ${ }^{1,2,3,4} \cdot$ S. García Mateo ${ }^{1,2} \cdot$ S. Hermoso-Durán² $\cdot$ D. Abad ${ }^{2} \cdot$ P. Carrera-Lasfuentes ${ }^{2,4}$. \\ A. Velazquez-Campoy ${ }^{2,3,4,5} \cdot$ O. Abian Franco ${ }^{2,3,4,6} \cdot$ A. Lanas La, $^{1,3,4}$
}

Published online: 6 July 2021

○) Springer-Verlag GmbH Germany, part of Springer Nature 2021

Correction to: International Journal of Colorectal Disease
https://doi.org/10.1007/s00384-021-03947-1

In the original published version of this article, the affiliations of the co-authors S. García Mateo and A. Lanas were updated.

S. García Mateo affiliation should be "Department of Gastroenterology, Lozano Blesa Clinic University Hospital, 50009 Zaragoza, Spain” and "Aragón Health Research Institute (IIS Aragón), 50009 Zaragoza, Spain" and the additional affiliation of A. Lanas should be "Department of Gastroenterology, Lozano Blesa Clinic University Hospital, 50009 Zaragoza, Spain”.

The original article has been corrected.

The original article can be found online at https://doi.org/10.1007/ s00384-021-03947-1.

S. García Mateo

sgarciamateo7@gmail.com

1 Department of Gastroenterology, Lozano Blesa Clinic

University Hospital, 50009 Zaragoza, Spain

2 Aragón Health Research Institute (IIS Aragón), 50009 Zaragoza, Spain

3 University of Zaragoza, 50009 Zaragoza, Spain

4 CIBERehd, 28029 Madrid, Spain

5 ARAID Foundation, Government of Aragon, Zaragoza, Spain

6 Aragon Institute of Health Science (IACS), 50009 Zaragoza, Spain 